\title{
ON FREE EXTERIOR POWERS
}

\author{
BY \\ HARLEY FLANDERS( $\left.{ }^{(}\right)$
}

1. Introduction. We study modules over a commutative ring $\boldsymbol{R}$ with unity. We seek properties of a module $\boldsymbol{M}$ for which some exterior power $\wedge^{p} \boldsymbol{M}$ is free of finite rank one or finitely generated.

This work originated in discussions with Howard Osborn. Several of his conjectures are settled below.

2. Preliminaries. Let $\boldsymbol{R}$ be a commutative ring with unity and let $\boldsymbol{M}$ be an $\boldsymbol{R}$ module (always assumed unital: $1 x=x$ for $x \in \boldsymbol{M}$ ).

Lemma 1. Suppose $\bigwedge^{n} M=0$. Then

$$
\bigwedge^{n+p} M=\mathbf{0} \text { for } p=1,2, \ldots
$$

Proof. The map

$$
\left(x_{1} \wedge \cdots \wedge x_{n}, y_{1} \wedge \cdots \wedge y_{p}\right) \rightarrow x_{1} \wedge \cdots \wedge y_{p}
$$

induces a surjection

$$
\left(\bigwedge^{n} \boldsymbol{M}\right) \otimes\left(\bigwedge^{p} M\right) \rightarrow \bigwedge^{n+p} \boldsymbol{M}
$$

The conclusion is immediate.

Lemma 2. Let $\boldsymbol{S}=\left(s_{1}, \ldots, s_{n}\right)$ and $\boldsymbol{T}$ be ideals of $\boldsymbol{R}$ such that $\boldsymbol{S}+\boldsymbol{T}=\boldsymbol{R}$. Then for each $p$,

$$
\left(s_{1}^{p}, \ldots, s_{n}^{p}\right)+\boldsymbol{T}=\boldsymbol{R} .
$$

This is proved by induction starting with the standard fact in commutative ideal theory that $S_{1}+T=S_{2}+T=R$ implies $S_{1} S_{2}+T=R$.

3. Vanishing of powers. The following result was conjectured by $\mathbf{H}$. Osborn in the free case.

THeOREM 1. Suppose for some $p$ that $\bigwedge^{p} \boldsymbol{M}$ is a cyclic $\boldsymbol{R}$-module. Then $\bigwedge^{p+k} \boldsymbol{M}=\mathbf{0}$ for $k=1,2, \ldots$.

Proof. It suffices to prove that $\bigwedge^{p+1} \boldsymbol{M}=\mathbf{0}$. Let $e$ be a generator of $\bigwedge^{p} \boldsymbol{M}$ and let $\boldsymbol{T}$ denote the ideal of annihilators of $\wedge^{p} \boldsymbol{M}$, i.e., annihilators of $e$. Then

$$
e=\sum_{1}^{n} x_{i 1} \wedge \cdots \wedge x_{i p}, \quad x_{i j} \in M
$$

Received by the editors December 23, 1968 and, in revised form, May 22, 1969.

( $\left.{ }^{1}\right)$ This research was supported by National Science Foundation Grant GP 6388. Reproduction for any purpose by the United States Government is permitted.

Copyright (C) 1969, American Mathematical Society 
But $x_{i 1} \wedge \cdots \wedge x_{i p}=s_{i} e, s_{i} \in R$; hence $\sum s_{i} e=e$. It follows that $\boldsymbol{S}+\boldsymbol{T}=\boldsymbol{R}$ where $\boldsymbol{S}=\left(s_{1}, \ldots, s_{n}\right)$.

Now let $y_{0} \wedge \cdots \wedge y_{p} \in \wedge^{p+1} M$, where $y_{j} \in M$. If $t \in T$, then

$$
t\left(y_{0} \wedge \cdots \wedge y_{p}\right)=y_{0} \wedge\left[t\left(y_{1} \wedge \cdots \wedge y_{p}\right)\right]=0 .
$$

Hence, by Lemma 2, we shall have proved that $y_{0} \wedge \cdots \wedge y_{p}=0$ once we have shown that $s_{i}^{2}\left(y_{0} \wedge \cdots \wedge y_{p}\right)=0$. It suffices to consider $i=1$. Now

$$
y_{1} \wedge \cdots \wedge y_{p}=r e \text { and } y_{0} \wedge x_{11} \wedge \cdots \wedge x_{1, p-1}=r^{\prime} e,
$$

hence

$$
\begin{aligned}
s_{1}^{2}\left(y_{0} \wedge \cdots \wedge y_{p}\right) & =s_{1}^{2} r\left(y_{0} \wedge e\right) \\
& =s_{1} r\left(y_{0} \wedge x_{11} \wedge \cdots \wedge x_{1 p}\right) \\
& =s_{1} r r^{\prime}\left(e \wedge x_{1 p}\right) \\
& =r r^{\prime}\left(x_{11} \wedge \cdots \wedge x_{1 p} \wedge x_{1 p}\right) \\
& =0
\end{aligned}
$$

The natural generalization of this result is the conjecture that if $\bigwedge^{p} \boldsymbol{M}$ can be generated by $q$ elements, then $\bigwedge^{p+q} \boldsymbol{M}=\mathbf{0}$. It is doubtful that this is true; however, we do have the following.

THEOREM 2. Suppose for some value of $p$ that $\wedge^{p} M$ is a free module of finite rank q. Then $\wedge^{p+q} \boldsymbol{M}=\mathbf{0}$.

Proof. Let $e_{1}, \ldots, e_{q}$ be a basis of $\bigwedge^{p} M$. Then

$$
e_{i}=\sum_{j} x_{i j}, \quad x_{i j}=x_{i j 1} \wedge \cdots \wedge x_{i j p}
$$

and

$$
x_{i j}=\sum s_{i j k} e_{k}, \quad s_{i j k} \in \boldsymbol{R}
$$

Hence

$$
e_{i}=\sum_{k}\left(\sum_{j} s_{i j k}\right) e_{k}, \quad \sum_{j} s_{i j k}=\delta_{i k}
$$

Here $1 \leqq i, k \leqq q$. Now select any $\left(j_{1}, j_{2}, \ldots, j_{q}\right)=j$. By Cramer's rule,

$$
\left[\operatorname{det}\left(s_{i j_{i} k}\right)_{i, k}\right] e_{r} \in \sum_{m=1}^{q} \boldsymbol{R} x_{m j_{m}} \text {. }
$$

Call the determinant factor $\Delta_{j}$. These determinants together generate the unit ideal $\boldsymbol{R}$ since

$$
1=\operatorname{det}\left(\delta_{i j}\right)=\operatorname{det}\left(\sum_{j} s_{i j k}\right)_{i, k}=\sum \Delta_{j}
$$


Renumbering, we have obtained quantities $t_{1}, t_{2}, \ldots \in R$ such that

(i) $\left(t_{1}, t_{2}, \ldots\right)=R$,

(ii) for each $i$,

$$
t_{i} \wedge^{p} \boldsymbol{M} \leqq \sum_{k=1}^{q} R y_{i k}
$$

where the $y_{i k}$ are pure $p$-vectors. From (i) and Lemma 2 above, $\left(t_{1}^{q+1}, t_{2}^{q+1}, \ldots\right)=\boldsymbol{R}$. We shall prove that

$$
t_{i}^{q+1}\left(\bigwedge^{p+q} M\right)=\mathbf{0} \quad(i=1,2, \ldots),
$$

which implies $\bigwedge^{p+q} \boldsymbol{M}=\mathbf{0}$. It suffices to prove

$$
t^{q+1}\left(\bigwedge^{p+q} M\right)=0
$$

if $t\left(\bigwedge^{p} M\right)$ is contained in a submodule of $\bigwedge^{p} M$ generated by $q$ pure $p$-vectors $y_{i}=y_{i 1} \wedge \cdots \wedge y_{i p}(i=1, \ldots, q)$. We resort to "abuse of language" to save notation in the calculation that follows:

$$
\begin{aligned}
t^{q+1}\left(\bigwedge^{p+q} M\right) & =t^{q+1}\left(\bigwedge^{p} M\right) \wedge\left(\wedge^{q} M\right) \\
& \leqq t^{q} \sum R y_{i} \wedge\left(\wedge^{q} M\right)=t^{q} \sum R y_{i 1} \wedge\left(\bigwedge^{p} M\right) \wedge\left(\bigwedge^{q-1} M\right) \\
& \leqq t^{q-1} \sum R y_{i 1} \wedge y_{j} \wedge\left(\wedge^{q-1} M\right) \\
& =t^{q-1} \sum R y_{i 1} \wedge y_{j 1} \wedge\left(\bigwedge^{p} M\right) \wedge\left(\bigwedge^{q-2} M\right) \\
& \leqq \cdots=\cdots \\
& \leqq \sum R y_{i 1} \wedge y_{j 1} \wedge \cdots \wedge y_{k 1} .
\end{aligned}
$$

But each $(q+1)$-tuple of indices $(i, j, \ldots, k)$ contains a repetition, hence the last module written vanishes. This completes the proof.

Besides the conjecture mentioned before the statement of the theorem, one might also ask whether

$$
\bigwedge^{q+1}\left(\bigwedge^{p} M\right)=\mathbf{0} \quad(p \geqq 1, q \geqq 0)
$$

implies

$$
\bigwedge^{p+q} M=\mathbf{0}
$$

4. Duality. Let $\boldsymbol{M}$ be an $\boldsymbol{R}$-module, and denote its conjugate space by $\boldsymbol{M}^{*}=$ $\operatorname{Hom}(\boldsymbol{M}, \boldsymbol{R})$. Recall the natural homomorphisms:

$$
\bigwedge^{p} M^{*} \rightarrow\left(\bigwedge^{p} M\right)^{*}, \quad M \rightarrow\left(M^{*}\right)^{*}
$$

The first is based on the pairing

$$
\left\langle f_{1} \wedge \cdots \wedge f_{p}, z_{1} \wedge \cdots \wedge z_{p}\right\rangle=\operatorname{det}\left(\left\langle f_{i}, z_{j}\right\rangle\right)
$$

of

$$
\left(\bigwedge^{p} M^{*}\right) \times\left(\bigwedge^{p} M\right) \rightarrow R
$$


If $\wedge^{p} M$ is free of rank one with basis $e$, then there are further natural (relative to $e$ ) homomorphisms:

$$
\bigwedge^{r} M \rightarrow\left(\bigwedge^{p-r} M\right)^{*} \quad(r=0, \ldots, p)
$$

based on

$$
\left(z_{1} \wedge \cdots \wedge z_{r}\right) \wedge\left(z_{r+1} \wedge \cdots \wedge z_{p}\right)=f\left(z_{r+1} \wedge \cdots \wedge z_{p}\right) e .
$$

The main result of this section, the theorem below, settles several conjectures of H. Osborn.

THEOREM 3. Let $\boldsymbol{M}$ be an $\boldsymbol{R}$-module such that $\bigwedge^{p} \boldsymbol{M}$ is free of rank one for some value of $p$. Then the following hold:

(i) $\boldsymbol{M}$ and $\boldsymbol{M}^{*}$ are finitely generated.

(ii) $\wedge^{p} M^{*}$ is free of rank one.

(iii) The natural map $\bigwedge^{p} M^{*} \rightarrow\left(\bigwedge^{p} M\right)^{*}$ is an isomorphism.

(iv) $\boldsymbol{M}$ is reflexive: the natural map $\boldsymbol{M} \rightarrow \boldsymbol{M}^{* *}$ is an isomorphism.

(v) For each $r=0, \ldots, p$, the natural map $\wedge^{r} M^{*} \rightarrow\left(\bigwedge^{r} M\right)^{*}$ is an isomorphism.

In addition, each module $\wedge^{r} \boldsymbol{M}, \wedge^{r} \boldsymbol{M}^{*}$ is reflexive:

$$
\bigwedge^{r} M \approx\left(\bigwedge^{r} M\right)^{* *}, \quad \bigwedge^{r} M^{*} \approx\left(\bigwedge^{r} M^{*}\right)^{* *}
$$

(vi) For each $r=0, \ldots, p$, the natural map (relative to a basis of $\wedge^{p} M$ )

$$
\bigwedge^{r} M \rightarrow\left(\bigwedge^{p-r} M\right)^{*}
$$

is an isomorphism.

(vii) The modules $\boldsymbol{M}$ and $\boldsymbol{M}^{*}$ are projective.

The proof will involve several steps.

Lemma 3. If $F \in\left(\bigwedge^{p} M\right)^{*}$ and $z_{0}, \ldots, z_{p} \in M$, then

$$
\sum(-1)^{j} F\left(z_{0} \wedge \cdots \wedge z_{j-1} \wedge z_{j+1} \wedge \cdots \wedge z_{p}\right) z_{j}=0 .
$$

Proof. The mapping

$$
\left(z_{0}, \ldots, z_{p}\right) \rightarrow \sum(-1)^{j} F\left(z_{0} \wedge \cdots \wedge z_{j-1} \wedge z_{j+1} \wedge \cdots \wedge z_{p}\right) z_{j}
$$

is alternating multilinear on $\times^{p+1} \boldsymbol{M} \rightarrow \boldsymbol{M}$, hence vanishes identically by Theorem 1.

For the next steps it is convenient to fix certain elements of $R, M, M^{*}$, and their exterior powers.

Let $e$ be a basis of $\wedge^{p} M$. Then $e=\sum_{1}^{n} b_{i} x_{i}$, where $b_{i} \in R, x_{i}=x_{i 1} \wedge \cdots \wedge x_{i p}$. Since $e$ is a basis, $x_{i}=s_{i} e, \sum b_{i} s_{i}=1$. Set

$$
y_{i j}=(-1)^{j-1} x_{i 1} \wedge \cdots \wedge x_{i, j-1} \wedge x_{i, j+1} \wedge \cdots \wedge x_{i p} \in \wedge^{p-1} M,
$$

so $x_{i}=x_{i j} \wedge y_{i j}$. Define $f_{i j} \in M^{*}$ by

$$
\left\langle f_{i j}, z\right\rangle e=z \wedge y_{i j} \quad(1 \leqq i \leqq n, 1 \leqq j \leqq p) .
$$


Then $\left\langle f_{i j}, x_{i j}\right\rangle=s_{i},\left\langle f_{i j}, x_{i k}\right\rangle=0(k \neq j)$. Set $f_{i}=f_{i 1} \wedge \cdots \wedge f_{i p} \in \bigwedge^{p} M^{*}$. Then

$$
\left\langle f_{i}, x_{i}\right\rangle=\operatorname{det}\left(\left\langle f_{i}, x_{i k}\right\rangle\right)_{j, k}=s_{i}^{p} .
$$

Also by a simple determinant computation, $\left\langle f_{i}, z \wedge y_{i j}\right\rangle=s_{i}^{p-1}\left\langle f_{i j}, z\right\rangle$.

The key to the whole proof is the following

Lemma 4. For $z \in M$ and for $g \in M^{*}$ we have

$$
\begin{aligned}
s_{i} z & =\sum_{j=1}^{p}\left\langle f_{i j}, z\right\rangle x_{i j}, \\
s_{i} g & =\sum_{j=1}^{p}\left\langle g, x_{i j}\right\rangle f_{i j}
\end{aligned}
$$

Proof. Since $\bigwedge^{p} M$ is free with basis $e$, there is an $F \in\left(\bigwedge^{p} M\right)^{*}$ such that $F(e)=1$. Apply Lemma 3 to $F ; z, x_{i 1}, \ldots, x_{i p}$ :

$$
F\left(x_{i}\right) z=\sum_{j} F\left(z \wedge y_{i j}\right) x_{i j} .
$$

But $F\left(x_{i}\right)=F\left(s_{i} e\right)=s_{i}$ and $F\left(z \wedge y_{i j}\right)=F\left(\left\langle f_{i j}, z\right\rangle e\right)=\left\langle f_{i j}, z\right\rangle$, so the first relation follows.

Now apply $g$ to this relation:

$$
s_{i}\langle g, z\rangle=\sum_{j}\left\langle f_{i j}, z\right\rangle\left\langle g, x_{i j}\right\rangle .
$$

Since this is true for all $z \in M$, the second relation follows.

Lemma 5. If $z \in M$ and if $g \in M^{*}$, then

$$
z=\sum_{i, j} b_{i}\left\langle f_{i j}, z\right\rangle x_{i j}, \quad g=\sum_{i, j} b_{i}\left\langle g, x_{i j}\right\rangle f_{i j} .
$$

Proof. Multiply each relation in Lemma 4 by $b_{i}$ and sum.

Proof of (i), Theorem 3. By Lemma 5 , the $x_{i j}(1 \leqq i \leqq n, 1 \leqq j \leqq p)$ span $M$ and the $f_{i j}$ span $M^{*}$.

By Lemma $2,\left(s_{1}^{p}, \ldots, s_{n}^{p}\right)=\boldsymbol{R}$. Hence there are $a_{i} \in \boldsymbol{R}$ satisfying

$$
\sum_{1}^{n} a_{i} s_{i}^{p}=1 .
$$

Set

$$
f=\sum_{1}^{n} a_{i} s_{i} f_{i} \in \bigwedge^{p} M^{*} .
$$

LEMMA 6. $s_{i}\left\langle f_{i}, e\right\rangle=s_{i}^{p},\langle f, e\rangle=1$.

Proof.

$$
\begin{gathered}
s_{i}\left\langle f_{i}, e\right\rangle=\left\langle f_{i}, s_{i} e\right\rangle=\left\langle f_{i}, x_{i}\right\rangle=s_{i}^{p}, \\
\langle f, e\rangle=\sum a_{i} s_{i}\left\langle f_{i}, e\right\rangle=\sum a_{i} s_{i}^{p}=1 .
\end{gathered}
$$


Lemma 7. For each $g \in \bigwedge^{p} M^{*}, g=\langle g, e\rangle f$.

Proof. It suffices to prove this for $g$ pure, $g=g_{1} \wedge \cdots \wedge g_{p}$. By Lemma 4, $s_{i} g_{k}=\sum_{j}\left\langle g_{k}, x_{i j}\right\rangle f_{i j}$. Multiply these together:

$$
s_{i}^{p} g=\left\langle g, x_{i}\right\rangle f_{i}=\langle g, e\rangle s_{i} f_{i} .
$$

Multiply by $a_{i}$ and sum to complete the proof.

LEMMA 8. For each $i, s_{i} f_{i}=s_{i}^{p} f$.

Proof. $s_{i} f_{i}=\left\langle s_{i} f_{i}, e\right\rangle f=\left\langle f_{i}, s_{i} e\right\rangle f=\left\langle f_{i}, x_{i}\right\rangle f=s_{i}^{p} f$.

Proof of (ii), Theorem 3. By Lemma 7, $f$ spans $\wedge^{p} M^{*}$. By Lemma 6 , if $c f=0$, then $c=c\langle f, e\rangle=0$, hence $f$ is free, $\wedge^{p} M^{*}$ is free of rank one with basis $f$.

Proof of (iii), Theorem 3. By Lemma $6, f \rightarrow$ generator of $\left(\bigwedge^{p} M\right)^{*}$, so the map is an isomorphism.

LemMA 9. The natural map $\boldsymbol{M} \rightarrow \boldsymbol{M}^{* *}$ is injective.

Proof. Suppose $z \in M$ and $g(z)=0$ for all $g \in M^{*}$. By the first formula in Lemma 5, $z=0$.

To complete the proof of (iv) we must study the homomorphisms of $\boldsymbol{M}^{*}$ induced by the $x_{i j}$. Let

$$
x_{i j} \rightarrow \phi_{i j} \in M^{* *} \quad \phi_{i j}(g)=\left\langle g, x_{i j}\right\rangle .
$$

LEMMA 10. For each $\phi \in M^{* *}$,

$$
\begin{aligned}
s_{i} \phi & =\sum_{j=1}^{p} \phi\left(f_{i j}\right) \phi_{i j} \quad(1 \leqq i \leqq n), \\
\phi & =\sum_{i, j} b_{i} \phi\left(f_{i j}\right) \phi_{i j} .
\end{aligned}
$$

Proof. Let $g \in M^{*}$. Apply $\phi$ to the second formula of Lemma 4:

$$
s_{i} \phi(g)=\sum_{j}\left\langle g, x_{i j}\right\rangle \phi\left(f_{i j}\right)=\sum_{j} \phi\left(f_{i j}\right) \phi_{i j}(g) .
$$

Hence the first formula of Lemma 10; the second formula easily follows.

Proof of (iv), Theorem 3. The map $\boldsymbol{M} \rightarrow \boldsymbol{M}^{* *}$ is injective by Lemma 9 and surjective by Lemma 10 (since $x_{i j} \rightarrow \phi_{i j}$ ), hence is an isomorphism.

Now we prepare for the proofs of (v) and (vi). The cases $r=0, r=p$ are trivial. Fix $r, 1 \leqq r \leqq p-1$. As before, let $H$ run over $r$-element subsets of $\{1, \ldots, p\}$ and let $H^{\prime}$ denote the complement of $H$. Set

$$
x_{i H}=x_{i h_{1}} \wedge \cdots \wedge x_{i h_{r}} \in \wedge^{r} M, \text { etc. }
$$

LEMMA 11. If $z \in \wedge^{r} M$, then

$$
s_{i}^{r} z=\sum_{H}\left\langle f_{i H}, z\right\rangle x_{i H} .
$$


If $g \in \wedge^{r} M^{*}$, then

$$
s_{i}^{r} g=\sum_{H}\left\langle g, x_{i H}\right\rangle f_{i H}
$$

both relations for $1 \leqq i \leqq n$.

These formulas follow easily from Lemma 4 applied to the special cases of $z, g$ pure.

Lemma 12. The $x_{i H}$ span $\wedge^{r} M$ and the $f_{i H}$ span $\wedge^{r} M^{*}$.

This follows from Lemma 11 and the consequences of Lemma 2: $\left(s_{1}^{r}, \ldots, s_{n}^{r}\right)=\boldsymbol{R}$.

Proof of (v), Theorem 3. Each $g \in \wedge^{r} M^{*}$ induces an element of $\left(\bigwedge^{r} M\right)^{*}$ via $z \rightarrow\langle g, z\rangle$. The resulting map $\bigwedge^{r} M^{*} \rightarrow\left(\bigwedge^{r} M\right)^{*}$ is injective. For suppose $\langle g, z\rangle=0$ for all $z$. By Lemma $11, s_{i}^{r} g=0$ for $1 \leqq i \leqq n$. But $R=\left(s_{1}^{r}, \ldots, s_{n}^{r}\right)$, hence $g=0$.

The map is also surjective. For let $F \in\left(\bigwedge^{r} M\right)^{*}$. Apply $F$ to the first formula of Lemma 11:

$$
s_{i}^{r} F(z)=\sum_{H}\left\langle f_{i H}, z\right\rangle F\left(x_{i H}\right) .
$$

Select $c_{i}$ so $\sum c_{i} s_{i}^{r}=1$. Then

$$
F(z)=\sum_{i, H} c_{i} F\left(x_{i H}\right)\left\langle f_{i H}, z\right\rangle,
$$

which shows that $F$ lies in the image of $\wedge^{r} M^{*}$. Hence the map is an isomorphism.

Similar reasoning, applied to the second identity of Lemma 11 , shows that each linear functional on $\wedge^{r} \boldsymbol{M}^{*}$ is induced by a unique element of $\wedge^{r} \boldsymbol{M}$, so each module is the conjugate of the other.

Proof of (vi), Theorem 3. We are considering the pairing

$$
\pi: \bigwedge^{r} \boldsymbol{M} \times \bigwedge^{p-r} \boldsymbol{M} \rightarrow \boldsymbol{R}
$$

given by $z \wedge w=\pi(z, w) e$, which induces

$$
\bigwedge^{r} \boldsymbol{M} \rightarrow\left(\bigwedge^{p-r} \boldsymbol{M}\right)^{*} \rightarrow \bigwedge^{p-r} \boldsymbol{M}^{*}
$$

Statement (vi) asserts that this map is an isomorphism.

It is an injection. Suppose $z \rightarrow 0$ for some $z \in \wedge^{r} M$, which means $z \wedge w=0$ for all $w \in \bigwedge^{p-r} M$. By Lemma 11 ,

Hence in particular,

$$
s_{i}^{r} z=\sum_{H}\left\langle f_{i H}, z\right\rangle x_{i H} .
$$

$$
\sum_{H}\left\langle f_{i H}, z\right\rangle x_{i H} \wedge x_{i K^{\prime}}=0,
$$

where $|K|=r$. Thus for each $H$, and each $i$,

$$
\begin{aligned}
& \left\langle f_{i H}, z\right\rangle x_{i}=0, \\
& s_{i}\left\langle f_{i H}, z\right\rangle=0 .
\end{aligned}
$$


To prove $z=0$, we shall show that the $s_{i} f_{i H}$ span $\wedge^{r} M^{*}$ and appeal to conclusion (v) of the theorem. But the second formula of Lemma 11 implies that if $g \in \wedge^{r} M^{*}$, then $s_{i}^{r+1} g$ is a linear combination of $s_{i} f_{i H}$. Since $\left(s_{1}^{r+1}, \ldots, s_{n}^{r+1}\right)=R$, so is $g$.

Now let $F \in\left(\bigwedge^{p-r} M\right)^{*}$. If $w \in \bigwedge^{p-r} M$, then (Lemma 11)

$$
s_{i}^{p-r} w=\sum_{K}\left\langle f_{i K^{\prime}}, w\right\rangle x_{i K^{\prime}}
$$

Hence

$$
s_{i}^{p-r} F(w)=\sum_{H}\left\langle f_{i H^{\prime}}, w\right\rangle F\left(x_{i H^{\prime}}\right)
$$

on the one hand, showing that the functionals $w \rightarrow s_{i}\left\langle f_{i H^{\prime}}, w\right\rangle \operatorname{span}\left(\bigwedge^{p-r} \boldsymbol{M}\right)^{*}$; and

$$
s_{i}^{p-r} x_{i H} \wedge w=\varepsilon_{H, H^{\prime}}\left\langle f_{i H^{\prime}}, w\right\rangle s_{i} e,
$$

or $s_{i}^{p-r} \pi\left(x_{i H}, w\right)=\varepsilon_{H, H^{\prime}} s_{i}\left\langle f_{i H^{\prime}}, w\right\rangle$ on the other hand. This latter relation shows that each $w \rightarrow s_{i}\left\langle f_{i H^{\prime}}, w\right\rangle$ is the image of $\pm s_{i}^{p-r} x_{i H}$; the mapping is surjective.

Proof of (vii), Theorem 3. Suppose we have homomorphisms

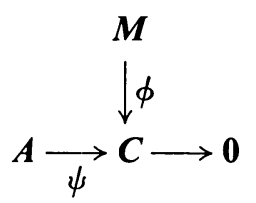

where the row is exact. Select $u_{i j} \in \boldsymbol{A}$ so $\psi\left(u_{i j}\right)=\phi\left(x_{i j}\right)$. Define $\lambda: \boldsymbol{M} \rightarrow \boldsymbol{A}$ by

$$
\lambda(z)=\sum b_{i}\left\langle f_{i j}, z\right\rangle u_{i j}
$$

By Lemma $5, \psi \circ \lambda=\phi$. Hence $\boldsymbol{M}$ is projective; similarly $\boldsymbol{M}^{*}$ is so.

REMARK. The isomorphism

$$
\bigwedge^{r} \boldsymbol{M} \rightarrow \bigwedge^{p-r} M^{*}
$$

can be made explicit by use of interior products. Indeed, as Professor Osborn has pointed out, this provides an alternate proof of (v). The products $\downarrow$ and $L$ are defined by

$$
\langle f\lrcorner z, w\rangle=\langle f, z \wedge w\rangle
$$

for $z \in \bigwedge^{r} M, w \in \bigwedge^{p-r} M$,

$$
\langle g, h\llcorner e\rangle=\langle h \wedge g, e\rangle
$$

for $h \in \bigwedge^{p-r} M^{*}, g \in \bigwedge^{r} M^{*}$. Thus

$$
\begin{aligned}
f\lrcorner: \bigwedge^{r} M & \rightarrow \bigwedge^{p-r} M^{*}, \\
\left\llcorner e: \bigwedge^{p-r} M^{*}\right. & \rightarrow \bigwedge^{r} M .
\end{aligned}
$$

The basic relation is the Cauchy-Binet Formula:

$$
\langle f\lrcorner z, g\llcorner e\rangle=\langle g, z\rangle
$$

for $z \in \bigwedge^{r} M, g \in \bigwedge^{p-r} M^{*}$. 
This is proved by calculation for the generators $x_{i H}, f_{i K^{\prime}}$. An easy consequence is the following pair of formulas:

$$
\begin{aligned}
& (f\lrcorner z)\left\llcorner e=(-1)^{r(p-r)} z\right. \\
& f\lrcorner\left(h\llcorner e)=(-1)^{r(p-r)} h\right.
\end{aligned}
$$

for $z \in \bigwedge^{r} M, h \in \bigwedge^{p-r} M^{*}$. Hence $\left.z \rightarrow f\right\lrcorner z, h \rightarrow h\llcorner e$ are isomorphisms, inverses of each other up to sign.

5. Other results. The following special case of Theorem 3 has some interest.

THEOREM 4. Suppose $\bigwedge^{p} M$ is free of rank one and $\bigwedge^{p} \boldsymbol{M}=\boldsymbol{R} e$ where $e$ is a pure vector, $e=x_{1} \wedge \cdots \wedge x_{p}$. Then $M$ is a free module of rank $p$.

Proof. Proceed as above, but with $n=1, s_{1}=1$. Thus $\left\langle f_{i}, x_{j}\right\rangle=\delta_{i j}$ so $x_{1}, \ldots, x_{p}$ are linearly independent. We know they span (Lemma 5), hence they form a basis of $\boldsymbol{M}$.

The following result is due to Osborn, who used it to obtain part of Theorem 3 under stronger hypotheses.

TheOREM 5. Suppose $R$ is a local ring and $\bigwedge^{p} M$ is free of rank one. Then $M$ is free.

Proof. Use the notation of Theorem 3. Then $\wedge^{p} \boldsymbol{M}$ has basis $e=\sum b_{i} x_{i}$, where $x_{i}$ are pure $p$-vectors, and $x_{i}=s_{i} e$ with $\sum b_{i} s_{i}=1$. Since $R$ is a local ring and $\left(s_{1}, \ldots, s_{n}\right)=\boldsymbol{R}$, some $s_{i}$ is a unit, hence $x_{i}$ is a basis and Theorem 4 applies.

Lemma 3 above can be generalized in a way which might be useful.

THEOREM 6. Let $\bigwedge^{p} M$ be cyclic. Let

$$
F \in \operatorname{Hom}\left(\bigwedge^{r} M, N_{1}\right) \text { and } G \in \operatorname{Hom}\left(\bigwedge^{p-r+1} M, N_{2}\right) \text {. }
$$

Let $z_{0}, \ldots, z_{p} \in M$. Then

$$
\sum_{H} \varepsilon_{H, H^{\prime}} F\left(z_{H}\right) \otimes G\left(z_{H^{\prime}}\right)=0
$$

where $H$ runs over $r$ element subsets of $\{0,1, \ldots, p\}$,

$$
H=\left\{i_{1}<i_{2}<\cdots<i_{r}\right\}, \quad H^{\prime}=\left\{j_{0}<j_{1}<\cdots<j_{p-r}\right\}
$$

is the complement of $H$ in $\{0,1, \ldots, p\}$, and

$$
z_{H}=z_{i_{1}} \wedge z_{i_{2}} \wedge \cdots \wedge z_{i_{r}}, \quad z_{H^{\prime}}=z_{j_{0}} \wedge \cdots \wedge z_{j_{p-r}} .
$$

Proof. The map

is alternating multilinear on

$$
\left(z_{0}, \ldots, z_{p}\right) \rightarrow \sum_{H} \varepsilon_{H, H^{\prime}} F\left(z_{H}\right) \otimes G\left(z_{H^{\prime}}\right)
$$

$$
\times^{p+1} M \rightarrow\left(\bigwedge^{r} N_{1}\right) \otimes\left(\bigwedge^{p-r+1} N_{2}\right) .
$$

But $\bigwedge^{p+1} \boldsymbol{M}=\mathbf{0}$ by Theorem 1 , hence the map vanishes. 
Some of the obvious generalizations of Theorem 3, conclusion (i) are wrong. For example the $\boldsymbol{Z}$ module $\boldsymbol{M}=\boldsymbol{Q} / \boldsymbol{Z}$ is not finitely generated, but $\wedge^{2} \boldsymbol{M}=\mathbf{0}$. Thus in general $\wedge^{p} \boldsymbol{M}$ finitely generated does not imply $\boldsymbol{M}$ finitely generated. Also $\wedge^{p} \boldsymbol{M}$ finitely generated (or even generated by one element) does not imply $\wedge^{p} \boldsymbol{M}^{*}$ finitely generated. For example, let $\boldsymbol{k}$ be a field, $\boldsymbol{V}$ a (countably) infinite dimensional $\boldsymbol{k}$-space. Set $\boldsymbol{R}=\boldsymbol{k} \oplus \boldsymbol{V}$ with trivial multiplication in $\boldsymbol{V}$. Set $\boldsymbol{M}=\boldsymbol{R} / \boldsymbol{V} \approx \boldsymbol{k}$. Then $\boldsymbol{M}$ is a cyclic $R$-module. But $M^{*}=\operatorname{Hom}(\boldsymbol{M}, \boldsymbol{R}) \approx V$, so each $\bigwedge^{p} M^{*}$ is infinite dimensional.

In Theorem 2 it was shown that $\wedge^{p} \boldsymbol{M}$ free of rank $q$ implies $\wedge^{p+q} \boldsymbol{M}=\mathbf{0}$. Assuming, of course, $q \geqq 1$, does this imply $\wedge^{p} M^{*}$ free of rank $q$ and the duality situation of Theorem 3 ? This is difficult and will be postponed for later investigation.

6. Examples. Let $\boldsymbol{R}$ be the ring of real analytic functions on the $p$-sphere $\boldsymbol{S}^{p}$ in Euclidean $E^{p+1}$ defined by $x_{0}^{2}+\cdots+x_{p}^{2}=1$. Let $M$ be the module of real analytic differentiable one-forms on $S^{p}$. The $\wedge^{p} M$ is free of rank one with basis the element of area

$$
\sigma=\sum(-1)^{j} x_{j} d x_{0} \wedge \cdots \wedge d x_{j-1} \wedge d x_{j+1} \wedge \cdots \wedge d x_{p} .
$$

By Theorem 3, $\boldsymbol{M}$ is projective; but $\boldsymbol{M}$ is not free unless $\boldsymbol{S}^{p}$ is parallelizable, i.e., $p=1,3$, or 7 . Our work above shows that each real analytic one-form on $S^{p}$ can be written $\sum f_{i} d x_{i}$ where $f_{i} \in \boldsymbol{R}$. (This is not obvious because the elements of $\boldsymbol{M}$ are cross sections of the bundle of one-forms at all points of $\boldsymbol{S}^{p}$.) Similar remarks apply to any real analytic manifold which is orientable and is a submanifold of euclidean space.

An algebraic model may be constructed as follows. Let $\boldsymbol{S}$ be a ring and $\boldsymbol{R}=$ $S[r, s, t]$ subject to $r^{2}+s^{2}+t^{2}=1$. Let $M=R x+R y+R z$ subject to $r x+s y+t z=0$. Then $\wedge^{2} M$ has the basis $e=r y \wedge z+s z \wedge x+t x \wedge y$ so $\boldsymbol{M}$ is projective; but $\boldsymbol{M}$ is not free.

A similar example is obtained from the ring $\boldsymbol{R}=\boldsymbol{S}[a, b, c, r, s, t]$ subject to the single relation $a r+b s+c t=1$. The module is $\boldsymbol{M}=\boldsymbol{R} x+\boldsymbol{R} y+\boldsymbol{R} z$ with the generating relation $r x+s y+t z=0$. The element $e=a y \wedge z+b z \wedge x+c x \wedge y$ is a basis of $\wedge^{2} M$, so $\boldsymbol{M}$ is projective; but $\boldsymbol{M}$ is not free.

To see that $e$ is a basis, first observe by the defining relations that

$$
x \wedge y=t e, \quad y \wedge z=r e, \quad z \wedge x=s e,
$$

hence $e$ generates $\bigwedge^{2} \boldsymbol{M}$. Next the map $F: \times^{2} \boldsymbol{M} \rightarrow \boldsymbol{R}$ given by

$$
F\left(\alpha_{1} x+\alpha_{2} y+\alpha_{3} z, \beta_{1} x+\beta_{2} y+\beta_{3} z\right)=\left|\begin{array}{ccc}
\alpha_{1} & \alpha_{2} & \alpha_{3} \\
\beta_{1} & \beta_{2} & \beta_{3} \\
r & s & t
\end{array}\right|
$$

is well defined and is alternating bilinear, hence defines $F: \bigwedge^{2} M \rightarrow R$. But $F(e)=1$, hence $e$ is free.

The proof that $\boldsymbol{M}$ is not free is more complicated. 


\section{REFERENCES}

1. N. Bourbaki, "Algèbre multilinéaire", Chapter 3 of Algèbre, Hermann, Paris, 1948.

2. H. Flanders, Tensor and exterior powers, J. Algebra 7 (1967), 1-24.

PURdue UnIVERSITY,

LAFAYETTE, INDIANA 